\title{
AUTOMORPHISMS OF METABELIAN PRIME POWER ORDER GROUPS OF MAXIMAL CLASS
}

\author{
S. FOULADI ${ }^{\bowtie}$ and R. ORFI
}

(Received 2 July 2007)

\begin{abstract}
Let $G$ be a $p$-group of maximal class of order $p^{n}$. It is shown that the order of the group of all automorphisms of $G$ centralizing the Frattini quotient takes the maximum value $p^{2 n-4}$ if and only if $G$ is metabelian. A structure theorem is proved for the Sylow $p$-subgroup, $\operatorname{Aut}_{p}(G)$, of the automorphism group of $G$ when $G$ is metabelian. For $p=2, \operatorname{Aut}_{2}(G)$ is the full automorphism group of $G$. For $p=3$, we prove a structure theorem for the full automorphism group of $G$.
\end{abstract}

2000 Mathematics subject classification: primary 20D45, 20D15.

Keywords and phrases: finite -group, maximal class, metabelian group, automorphism group.

\section{Introduction}

There have been a number of studies of the automorphism groups of $p$-groups of maximal class (see for example, Baartmans and Woeppel [2], Juhász [8], Malinowska [10], Caranti and Mattarei [4], Caranti and Scoppola [5]). These concentrate mostly on small automorphism groups. In this paper we consider large automorphism groups.

Let $G$ be a $p$-group of maximal class of order $p^{n}$ and let $\Phi=\Phi(G)$ be the Frattini subgroup of $G$. It is well known [7, Satz III.3.17] that the order of $\operatorname{Aut}^{\Phi}(G)$, the group of all automorphisms of $G$ centralizing $G / \Phi$, divides $p^{2 n-4}$. Moreover, the order of $\operatorname{Aut}_{p}(G)$, the Sylow $p$-subgroup of the automorphism group of $G$, divides $p^{2 n-3}$. In Section 4 we prove that $\left|\operatorname{Aut}^{\Phi}(G)\right|$, the order of $\operatorname{Aut}^{\Phi}(G)$, is $p^{2 n-4}$ if and only if $G$ is metabelian (Theorem 4.3).

Juhász [8, Theorem 2.3] proved that if $G$ is a $p$-group of maximal class then $\operatorname{Aut}^{\Phi}(G)$ is a split extension of $\operatorname{Inn}(G)$, the inner automorphism group of $G$. In Section 3 we prove that when $G$ is metabelian, a complement of $\operatorname{Inn}(G)$ in $\operatorname{Aut}^{\Phi}(G)$ is almost homocyclic of rank $p-1$; that is, it is a direct product of exactly $p-1$ cyclic groups of order $p^{r}$ or $p^{r+1}$ for some nonnegative integer $r$ (Theorem 3.3). Also we give conditions on $G$ for $\left|\operatorname{Aut}_{p}(G)\right|=p^{2 n-3}$ (Corollary 3.8). In this case $\operatorname{Aut}_{p}(G)$

(C) 2008 Australian Mathematical Society 0004-9727/08 \$A2.00 + 0.00 
is a split extension of $\operatorname{Aut}^{\Phi}(G)$ by a cyclic group of order $p$ (Theorem 3.10). For $p=2$, the automorphism group is a 2 -group. In Section 5 we give a simple proof for the structure theorem in this case (Theorem 5.9). It is straightforward to see that when $p$ is odd, the (full) automorphism group $\operatorname{Aut}(G)$ of $G$ is a split extension of $\operatorname{Aut}_{p}(G)$ by a subgroup of the direct product of two cyclic groups of order $p-1$, see [2, Section 1]. By using this result we prove a structure theorem for $\operatorname{Aut}(G)$ when $p=3$ (Theorem 5.8).

Throughout this paper the following notation is used. The terms of the lower and the upper central series of $G$ are denoted by $\gamma_{i}(G)$ and $\zeta_{i}(G)$, respectively. The centre of $G$ is denoted by $Z=Z(G)$. The nilpotency class of a group $G$ is denoted by $\operatorname{cl}(G)$. If $\alpha$ is an automorphism of $G$ and $x$ is an element of $G$, we write $x^{\alpha}$ for the image of $x$ under $\alpha$. The inner automorphism induced by the element $g$ is denoted by $\sigma_{g}$. For a normal subgroup $N$ of $G$, we let $\operatorname{Aut}^{N}(G)$ denote the group of all automorphisms of $G$ centralizing $G / N$. Also $C_{n}$ denotes the cyclic group of order $n$. All unexplained notation is standard and follows that of [9].

\section{Some basic results}

In this section we give some basic results needed for the main results of the paper.

Let $G$ be a $p$-group of maximal class and order $p^{n}(n \geq 4)$, where $p$ is a prime. Following [9], we define the 2-step centralizer $K_{i}$ in $G$ to be the centralizer in $G$ of $\gamma_{i}(G) / \gamma_{i+2}(G)$ for $2 \leq i \leq n-2$ and define $P_{i}=P_{i}(G)$ by $P_{0}=G, P_{1}=K_{2}$, $P_{i}=\gamma_{i}(G)$ for $2 \leq i \leq n$. The degree of commutativity $l=l(G)$ of $G$ is defined to be the maximum integer such that $\left[P_{i}, P_{j}\right] \leq P_{i+j+l}$ for all $i, j \geq 1$ if $P_{1}$ is not Abelian and $l=n-3$ if $P_{1}$ is Abelian.

Take $s \in G-\bigcup_{i=2}^{n-2} K_{i}, s_{1} \in P_{1}-P_{2}$ and $s_{i}=\left[s_{i-1}, s\right]$ for $2 \leq i \leq n-1$. It is easily seen that $\left\{s, s_{1}\right\}$ is a generating set for $G$ and $P_{i}(G)=\left\langle s_{i}, \ldots, s_{n-1}\right\rangle$ for $1 \leq i \leq n-1$.

For the rest of the section we fix the above notation and assume that $n \geq 4$.

LEMMA 2.1 [9, Corollary 3.2.7]. Let $G$ be a p-group of maximal class. The degree of commutativity of $G$ is positive if and only if the 2-step centralizers of $G$ are all equal.

LEMMA 2.2 [7, Hilfssatz III. 14.13]. If $G$ is a p-group of maximal class of order $p^{n}$ and $s \notin K_{i}$ for $2 \leq i \leq n-2$, then $\mathcal{C}_{G}(s)=\langle s\rangle P_{n-1}(G)$ and $s^{p} \in P_{n-1}$.

LEMMA 2.3. Let $G$ be a p-group of maximal class of order $p^{n}$.

(i) If $G$ has positive degree of commutativity, then $s_{i}^{p} s_{i+p-1} \in P_{i+p}$ for $i>1$.

(ii) If $n>p+1$ then $s_{1}^{p} s_{p} \in P_{p+1}$.

(iii) If $y \in P_{2}$ then $(s y)^{p}=s^{p}$.

(iv) If $G$ is metabelian then $G$ has positive degree of commutativity.

(v) If $G$ is metabelian then $s_{i}^{p} s_{i+1}^{\left(\begin{array}{c}p \\ 2\end{array}\right)} \cdots s_{i+p-1}=1$ for $2 \leq i \leq n-1$. 
Proof. Conclusions (i)-(iv) follow by [9, Propositions 3.3.8, 3.3.3, Lemma 3.3.7] and [3, Corollary p. 59]. Conclusion (v) is obvious since $\left(s s_{i}\right)^{p}=s^{p} s_{i}^{p} s_{i+1}^{\left(\begin{array}{c}p \\ 2\end{array}\right)}$ $\cdots s_{i+p-1}$.

Lemma 2.4 [3, Theorem 3.10]. If $G$ is a metabelian p-group of maximal class of order $p^{n}(n \geq p+1)$, then $\left[P_{1}(G), P_{i}(G)\right] \leq P_{n-p+i}(G)$.

LEMMA 2.5. Let $G$ be a metabelian p-group of maximal class of order $p^{n}$.

(i) $s_{i}^{p^{t}}=s_{i+(p-1) t}^{(-1)^{t}} x$, where $x \in P_{i+(p-1) t+1}$ for $i \geq 2$; so if $s_{i}^{p^{t}} \neq 1$ then $s_{i}^{p^{t}} \in P_{i+(p-1) t}-P_{i+(p-1) t+1}$.

(ii) $P_{i}^{p^{t}} \leq P_{i+(p-1) t}$ for $i \geq 2$.

PROOF.

(i) We use induction on $t$ and Lemma 2.3(i).

(ii) This follows from (i).

LEMMA 2.6. Let $G$ be a metabelian p-group of maximal class of order $p^{n}$ and $m$ be a positive integer.

(i) $\left[s_{1}, s^{m}\right]=s_{2}^{m} s_{3}^{\left(\begin{array}{c}m \\ 2\end{array}\right)} \cdots s_{m+1}^{\left(\begin{array}{c}m \\ m\end{array}\right)}$.

(ii) $\left[s_{i}^{m}, s_{1}\right]=\left[s_{i}, s_{1}\right]^{m}$ for $i \geq 2$.

(iii) $\left[s_{i}^{m}, s\right]=\left[s_{i}, s\right]^{m}$ for $i \geq 2$.

(iv) $\left|s_{2}\right| \geq\left|s_{3}\right| \geq \cdots \geq\left|s_{n-1}\right|$ and so $\exp \left(P_{i}\right)=\left|s_{i}\right|$ for $i \geq 2$.

(v) $\left(s_{1} s_{i}\right)^{m}=s_{1}^{m} s_{i}^{m}\left[s_{i}, s_{1}\right]\left[s_{i}, s_{1}^{2}\right] \cdots\left[s_{i}, s_{1}^{m-1}\right]$ for $i \geq 2$.

LEMMA 2.7. Let $G$ be a metabelian p-group of maximal class of order $p^{n}$. Then

$$
\left|s_{i}\right|= \begin{cases}p^{((n-i) /(p-1))}, & p-1 \mid n-i, \\ p^{[(n-i) /(p-1)]+1}, & p-1 \nmid n-i,\end{cases}
$$

for $i \geq 2$.

PROOF. It is enough to prove that $\left|s_{n-i(p-1)-j}\right|=p^{i+1}$ for all $i \geq 0$ and all $1 \leq j$ $\leq p-1$. We use induction on $i$. It is easy to show that $\left|s_{n-j}\right|=p$ for $1 \leq j \leq p-1$, by Lemma 2.3(i). Now the result follows from Lemmas 2.3(i), 2.6(iv) and 2.5.

Lemma 2.8. Let $G$ be a metabelian p-group of maximal class of order $p^{n}$. Then $s_{r i-(r-1)}^{\left(\begin{array}{c}p_{r}^{t} \\ r\end{array}\right)} \in P_{i+2+t(p-1)}$ when $t \geq 0, r \geq 2$ and $i \geq 3$.

PROOF. Suppose that $r=p^{w} m$, where $(m, p)=1$ and $w \geq 0$. So $\left(\begin{array}{c}p^{t} \\ r\end{array}\right)=p^{t-w} k$, where $(k, p)=1$. Therefore $s_{r i-(r-1)}^{\left(\begin{array}{c}p^{t} \\ r\end{array}\right)} \in P_{r i-(r-1)+(t-w)(p-1)}$ by Lemma 2.5(i). We have the equality $p^{w}>w(p-1)$ for $w \geq 0$ and $p \geq 3$. Hence, $(i-1)(r-1)$ $\geq w(i-1)(p-1)$. Moreover, $\quad w(p-1)(i-1) \geq 2 w(p-1) \geq w(p-1)+2$ when $w>0$, and $(i-1)(r-1) \geq 2$ when $w=0$, completing the proof. 
LEMMA 2.9. Let $G$ be a metabelian p-group of maximal class of order $p^{n}$.

(i) Suppose that $i \geq 2$ and $1 \leq k \leq p-2$. If $s_{i}^{p^{t}}=s_{i+1}^{p^{m_{1}} u_{1}} \cdots s_{i+k}^{p^{m_{k}} u_{k}}$, where $\left(u_{j}, p\right)=1$ and $m_{j} \geq t-1$ for $1 \leq j \leq k$, then $s_{i}^{p^{t}}=1$.

(ii) $\left\langle s_{i}\right\rangle \cap\left\langle s_{i+1}, \ldots, s_{i+k}\right\rangle=1$ for $i \geq 2$ and $1 \leq k \leq p-2$.

(iii) $P_{i}=\left\langle s_{i}\right\rangle \times\left\langle s_{i+1}\right\rangle \times \cdots \times\left\langle s_{i+p-2}\right\rangle$ for $i \geq 2$.

(iv) Suppose that $n-r=(p-1) k+j$ for $0 \leq j \leq p-2, r \geq 2$. If $j \neq 1$, then $\left|Z s_{r}\right|=\left|s_{r}\right|$ and $\left|Z s_{r}\right|=\left|s_{r}\right| / p$ if $j=1$.

(v) $\quad P_{i} / Z \cong\left\langle Z s_{i}\right\rangle \times \cdots \times\left\langle Z s_{i+p-2}\right\rangle$ for $i \geq 2$.

ProOF. (i) We first note that if $m_{j} \geq t$ for all $j, 1 \leq j \leq k$, then by Lemma 2.5(i) $s_{i+j}^{p^{m} u_{j}} \in P_{i+t(p-1)+1}$ and so $s_{i}^{p^{t}}=1$. Now suppose that $j(1 \leq j \leq k)$ is the least positive integer such that $m_{j}=t-1$, so $m_{1}, m_{2}, \ldots, m_{j-1} \geq t$. We claim that $s_{i+j}^{p^{m_{j}} u_{j}}=1$. Suppose that this is false; then by Lemma 2.5(i) $s_{i+j}^{p^{m} u_{j}} \in P_{i+j+(t-1)(p-1)}-P_{i+j+(t-1)(p-1)+1}$. On the other hand, we see that $s_{r+i}^{p^{m_{r}} u_{r}} \in P_{i+j+(t-1)(p-1)+1}$ for $1 \leq r \leq k, \quad r \neq j$. Since $j \leq p-2$, we have $s_{i}^{p^{t}} \in P_{i+j+(t-1)}(p-1)+1$, which is impossible. Therefore, by the above note, the proof is established.

(ii) We use induction on $k$. By Lemma 2.7, $\left|s_{i+j}\right| \geq\left|s_{i}\right| / p$ for $1 \leq j$ $\leq p-2$. For $k=1$, we suppose that $s_{i}^{p^{t}} \in\left\langle s_{i}\right\rangle \cap\left\langle s_{i+1}\right\rangle$. We may write $s_{i}^{p^{t}}$ $=s_{i+1}^{p^{m} u}$, where $(u, p)=1$. By considering the order of both sides we conclude that $m \geq t-1$ so that $s_{i}^{p^{t}}=1$ by (i). Now suppose that $i \geq 2$ and the equality holds for all positive integers less than $k$. If $\left\langle s_{i}\right\rangle \cap\left\langle s_{i+1}, \ldots, s_{i+k}\right\rangle \neq 1$ then we may write $s_{i}^{p^{t}}=s_{i+1}^{p^{m} u_{1}} \cdots s_{i+k}^{p^{m_{k}} u_{k}}$, where $\left(u_{j}, p\right)=1$ for $1 \leq j \leq k$. Again by considering the order of both sides and using the induction hypothesis we deduce that $\left|s_{i}^{p^{t}}\right|=\max \left\{\left|s_{i+1}\right| / p^{m_{1}}, \ldots,\left|s_{i+k}\right| / p^{m_{k}}\right\}$. Hence $m_{j} \geq t-1$ for $1 \leq j \leq k$ and therefore the proof is completed by applying (i).

(iii) On setting $H_{i}=\left\langle s_{i}, \ldots, s_{i+p-2}\right\rangle$ we see that $H_{i} \leq P_{i}$; also by (ii), $H_{i} \cong\left\langle s_{i}\right\rangle \times \cdots \times\left\langle s_{i+p-2}\right\rangle$. Now by Lemma 2.7 we deduce that $\left|H_{i}\right|=p^{n-i}$ and hence $H_{i}=P_{i}$, as required.

(iv) This is proved by Lemma 2.5(i).

(v) We may proceed as in (iii) above.

Corollary 2.10. $P_{i}$ is an almost homocyclic p-group of rank $p-1$ for $i \geq 2$.

Proof. This follows from Lemma 2.9(iii) and the fact that $\left|P_{i}\right|=p^{n-i}$. Also we note that elementary Abelian groups of order $p, p^{2}, \ldots, p^{p-1}$ are almost homocyclic of rank $p-1$ with $r=0$, by our definition in the introduction and for each $n, d$ there is exactly one almost homocyclic group of order $p^{n}$ and rank $d$. 
LEMMA 2.11. Let $G$ be a group and $x, y$ be elements of $G$. If $\left[[x, y], x^{-1}\right]=1$ then $[x, y]=\left[y, x^{-1}\right]$.

LEMMA 2.12. Let $G$ be a metabelian p-group of maximal class of order $p^{n}$. If $n \geq 2 p-3, p \geq 3$ and $m$ is a positive integer, then:

(i) $\left[\left[s_{2}, s_{1}\right], s_{1}\right]=z \in\left[P_{p-1}, P_{1}\right]$ and $\left[\left[s_{i}, s_{1}\right], s_{1}\right]=1$ for $i \geq 3$, moreover $z=1$ if $n \geq 2 p-2$;

(ii) $\left[\left[s_{i}, s_{1}\right]^{m}, s\right]=\left[s_{i+1}, s_{1}\right]^{m}$ for $i \geq 2$;

(iii) $\left(s_{1} s_{2}\right)^{m}=s_{1}{ }^{m} s_{2}{ }^{m}\left[s_{2}, s_{1}\right]^{\left(\begin{array}{c}m \\ 2\end{array}\right)} z^{\left(\begin{array}{c}m \\ 3\end{array}\right) \text {; }}$

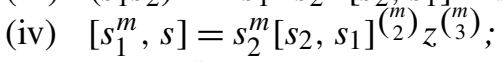

(v) $\left[s_{i}, s_{1}\right]^{p}=1$ for $i \geq 2$.

PROOF. (i) This follows from Lemma 2.4.

(ii) We use induction on $m$, the Witt identity, Lemma 2.11 and (i).

(iii)-(iv) We use induction on $m$, (i) and Lemma 2.6(ii).

(v) We have $\left[s_{i}, s_{1}\right] \in P_{n-p+i}$ by Lemma 2.4 and $\exp \left(P_{n-p+i}\right) \mid p$ by Lemma 2.5(ii).

LEMMA 2.13. We have $\sum_{k=u}^{m} k\left(\begin{array}{l}k \\ u\end{array}\right)=m\left(\begin{array}{l}m+1 \\ u+1\end{array}\right)-\left(\begin{array}{c}m+1 \\ u+2\end{array}\right)$ for all positive integers $u$ and $m$.

LEMMA 2.14. Let $G$ be a metabelian p-group of maximal class of order $p^{n}$. If $n \geq 2 p-3$ and $p \geq 3$, then $\left(s s_{1}\right)^{p}=s^{p} s_{1}^{\left(\begin{array}{l}p \\ 1\end{array}\right)} \ldots s_{p}^{\left(\begin{array}{c}p \\ p\end{array}\right)}\left[s_{1}, s_{p-1}\right]$.

PROOF. By using induction on $m$, Lemmas 2.13 and 2.12 we see that

$$
\left(s s_{1}\right)^{m}=s^{m} s_{1}^{\left(\begin{array}{c}
m \\
1
\end{array}\right)} \cdots s_{m}^{\left(\begin{array}{c}
m \\
m
\end{array}\right)}\left[s_{2}, s_{1}\right]^{b_{2}} \cdots\left[s_{i}, s_{1}\right]^{b_{i}} \cdots\left[s_{m}, s_{1}\right]^{b_{m}} z^{b_{3}},
$$

where $b_{i}=\sum_{k=i-1}^{m-1} k\left(\begin{array}{c}k \\ i-1\end{array}\right)$ for $i \geq 2$. Now we take $m=p$ and observe that $\left[s_{p}, s_{1}\right]=1$ by Lemma 2.4. We have $b_{i}=(p-1)\left(\begin{array}{c}p \\ i\end{array}\right)-\left(\begin{array}{c}p \\ i+1\end{array}\right)$ by Lemma 2.13, so $p \mid b_{i}$ when $i<p-1$ and $b_{p-1}=p(p-1)-1$. Therefore $\left[s_{i}, s_{1}\right]^{b_{i}}=1$ for $i<p-1$ and $\left[s_{p-1}, s_{1}\right]^{b_{p-1}}=\left[s_{1}, s_{p-1}\right]$ by Lemma 2.12(v). Also if $p=3$, then by Lemma 2.12(i), $z=1$ since $n \geq 4$; and if $p>3$, then $p \mid b_{3}$. Hence, $z^{b_{3}}=1$ since $z \in Z(G)$.

In what follows we give a presentation for a metabelian $p$-group $G$ of maximal class of order $p^{n}(n \geq 2 p-3)$. Suppose that $k$ is the largest positive integer such that $\left[P_{1}, P_{2}\right]=P_{k}$, so $k \geq n-p+2$ by Lemma 2.4. Therefore we may write $\left[s_{1}, s_{2}\right]=s_{k}^{a_{1}} s_{k+1}^{a_{2}} \cdots s_{n-1}^{a_{n-k}}$, where $a_{1} \neq 0$ and $o \leq a_{i}<p$. Also, by Lemma 2.2, $s^{p}=s_{n-1}^{w}(0 \leq w<p)$, and, by Lemma 2.3(v), $s_{i}^{p} s_{i+1}^{\left(\begin{array}{c}p \\ 2\end{array}\right)} \cdots s_{i+p-1}=1$ for $i \geq 2$. Now by Lemmas 2.14 and 2.4 we see that $s_{1}^{\left(\begin{array}{c}p \\ 1\end{array}\right)} \cdots s_{p}^{\left(\begin{array}{c}p \\ p\end{array}\right)}=s_{n-1}^{z}(0 \leq z<p)$. So we have proved the following theorem. 
THEOREM 2.15. Let $G$ be a metabelian p-group of maximal class of order $p^{n}, p \geq 3$ and $n \geq 2 p-3$. Then

$$
\begin{gathered}
G \cong\left\langle s, s_{1}, \ldots, s_{n-1}\right| s_{i}=\left[s_{i-1}, s\right],\left[s_{n-1}, s\right]=1,\left[s_{1}, s_{2}\right]=s_{k}^{a_{1}} s_{k+1}^{a_{2}} \cdots s_{n-1}^{a_{n-k}} \\
\left.\left[s_{i}, s_{j}\right]=1, s^{p}=s_{n-1}^{w}, s_{1}^{\left(\begin{array}{c}
p \\
1
\end{array}\right)} \cdots s_{p}^{\left(\begin{array}{c}
p \\
p
\end{array}\right)}=s_{n-1}^{z}, s_{i}^{p} s_{i+1}^{\left(\begin{array}{c}
p \\
2
\end{array}\right)} \cdots s_{i+p-1}=1\right\rangle,
\end{gathered}
$$

where $2 \leq i \leq j \leq n-1, a_{1} \neq 0,0 \leq a_{1}, \ldots, a_{n-k}<p, 0 \leq z<p$ and $0 \leq w<p$.

\section{3. $\operatorname{Aut}^{P_{i}}(G)$ and $\operatorname{Aut}_{p}(G)$}

In this section we prove a structure theorem for $\operatorname{Aut}^{P_{i}}(G)(i \geq 2)$ and $\operatorname{Aut}_{p}(G)$ when $G$ is a metabelian $p$-group of maximal class of order $p^{n}$. We note that if $n \leq 3$ and $G$ is not cyclic then $\operatorname{Aut}^{\Phi}(G)=\operatorname{Inn}(G)$ and $\operatorname{Aut}_{p}(G) \cong \operatorname{Aut}^{\Phi}(G) \rtimes C_{p}$. Moreover, when $G$ is cyclic then $\operatorname{Aut}_{p}(G)=\operatorname{Aut}^{\Phi}(G) \cong C_{p}$. Therefore, in the rest of this section we assume that $n \geq 4$.

Theorem 3.1 [6, Theorem 3.2]. Let $G=\langle a, b\rangle$ be a two-generated metabelian group. Then the following are equivalent:

(i) for all $u, v \in G^{\prime}$ there is an automorphism of $G$ that maps a to au and $b$ to bv;

(ii) $G$ is nilpotent.

By the above theorem we see that if $G$ is a noncyclic metabelian $p$-group of maximal class of order $p^{n}$, then for any elements $x, y \in G^{\prime}$ there is an automorphism that maps $s$ to $s x$ and $s_{1}$ to $s_{1} y$, so $\left|\operatorname{Aut}^{\Phi}(G)\right|=p^{2 n-4}$. Now we define $\alpha_{i}, 2 \leq i$ $\leq n-1$, by $s^{\alpha_{i}}=s$ and $s_{1}^{\alpha_{i}}=s_{1} s_{i}$. Clearly, $\left[\alpha_{i}, \alpha_{j}\right]=1$. Also $\alpha_{2}=\sigma_{s}$ has order $p$.

LEMMA 3.2. Let $G$ be a metabelian p-group of maximal class of order $p^{n}$. Then $\left|\alpha_{i}\right|=\left|s_{i}\right|$ for $i \geq 3$.

PROOF. We observe that $\left(s_{1}\right)^{\alpha_{i}^{m}}=s_{1} s_{i}^{m} s_{2 i-1}^{\left(\begin{array}{c}m \\ 2\end{array}\right)} \cdots s_{r i-(r-1)}^{\left(\begin{array}{c}m \\ r\end{array}\right)} \cdots s_{m i-(m-1)}^{\left(\begin{array}{c}m \\ m\end{array}\right)}$ for every positive integer $m$. On setting $m=\left|s_{i}\right|=p^{t}$, we see that $t \geq((n-i) /(p-1))$ by Lemma 2.7 , so $\alpha_{i}^{m}=1$ by Lemma 2.8. Also $\alpha_{i}^{p^{k}} \neq 1$ for $p^{k}<\left|s_{i}\right|$; otherwise $s_{i}^{p^{k}} s_{2 i-1}^{\left(p_{2}^{k}\right)} \cdots s_{r i-(r-1)}^{\left(\begin{array}{c}p^{k} \\ r\end{array}\right)} \cdots=1$. However, $s_{i}^{p^{k}} \in P_{i+k(p-1)}-P_{i+k(p-1)+1}$ by Lemma 2.5(i) and $s_{r i-(r-1)}^{\left(\begin{array}{c}p^{k} \\ r\end{array}\right)} \in P_{i+k(p-1)+2}$ for $2 \leq r \leq m$ by Lemma 2.8, which is impossible.

THEOREM 3.3. Let $G$ be a metabelian p-group of maximal class of order $p^{n}$. We set $A_{i}=\left\langle\alpha_{i}, \ldots, \alpha_{n-1}\right\rangle$ and $I_{i}=\left\{\sigma_{g} \mid g \in P_{i-1}\right\}$ for $2 \leq i \leq n-1$.

(i) $\operatorname{Aut}^{\Phi}(G)=\operatorname{Inn}(G) \rtimes A_{3}$, where $A_{3}$ is an almost homocyclic p-group of rank $p-1$.

(ii) $\operatorname{Aut}^{P_{i}}(G)=I_{i} \rtimes A_{i}$ for $2 \leq i \leq n-1$.

(iii) $A_{i} \cong I_{i} \cong P_{i}$ is an almost homocyclic p-group of rank $p-1$, having order $p^{n-i}$ for $i \geq 3$ and $A_{2} \nsucceq P_{2}$ when $n>p+1$. 
Proof. (i) By [8, Theorems 2.3, 4.3], $\operatorname{Aut}^{\Phi}(G)=\operatorname{Inn}(G) \rtimes A_{3}$ and $A_{3} \cong P_{3}$, so $A_{3}$ is almost homocyclic by Corollary 2.10 .

(ii) We have $\left|I_{i}\right|=p^{n-i}$ for $2 \leq i \leq n-1$ and, by Theorem 3.1, $\left|\operatorname{Aut}^{P_{i}}(G)\right|$ $=p^{2(n-i)}$ for $2 \leq i \leq n-1$. Also $A_{n-1}<\cdots<A_{3}<A_{2}$ implies that $\left|A_{i}\right| \geq p^{n-i}$. Therefore by (i), $\left|A_{i}\right|=p^{n-i}$ and $I_{i} \cap A_{i}=1$ for $3 \leq i \leq n-1$. So it remains to prove that $I_{2} \cap A_{2}=1$. Otherwise there exists an element $g \in P_{1}-Z(G)$ such that $\sigma_{g} \in A_{2}$ and so $[s, g]=1$. Hence by Lemma 2.2, $g=s^{i} z$ for some $0<i<p$ and some $z \in Z(G)$. Thus $\left[s_{1}, g\right]=\left[s_{1}, s^{i}\right]=s_{2}^{i} s_{3}^{\left(\begin{array}{c}i \\ 2\end{array}\right)} \cdots s_{i+1}^{\left(\begin{array}{c}i \\ i\end{array}\right)}$, by Lemma 2.6(i). It follows that $\left[s_{1}, g\right] \in P_{2}-P_{3}$. Since $g \in P_{1}$ and $G$ has positive degree of commutativity, we find that $\left[s_{1}, g\right] \in P_{3}$, which is a contradiction. Therefore, $I_{2} \cap A_{2}=1$.

(iii) We have $\left|A_{i}\right|=p^{n-i}$ by (ii). By [8, Theorem 4.3], $A_{i} \cong P_{i}$ for $i \geq 3$ and so, by Corollary 2.10, $A_{i}$ is almost homocyclic. Now we prove that $I_{i} \cong P_{i}$ for $i \geq 3$. To see this we note that $I_{i} \cong P_{i-1} / Z$. Also $P_{i-1} / Z \cong P_{i}$ by Lemma $2.9(\mathrm{v})$, (iv) and Corollary 2.10 we have $I_{i} \cong P_{i} \cong A_{i}$ for $i \geq 3$. Finally, $A_{2}=\left\langle\alpha_{2}\right\rangle A_{3}$ and $\alpha_{2}=\sigma_{s}$. Therefore $A_{2}=\left\langle\alpha_{2}\right\rangle \times A_{3}$ since $\sigma_{s}$ has order $p$. So for $n>p+1$ the minimal number of generators of $P_{2}$ and $A_{2}$ are different.

LEMMA 3.4. With the notation and assumption of Theorem 3.3, the following inequalities hold for all positive integers $m$.

(i) $\left[\sigma_{s_{t}}, \alpha_{k}\right]=\sigma_{s_{t+k-1}}$ for $2 \leq k \leq n-1$ and $1 \leq t \leq n-1$.

(ii) $\left[A_{k}, I_{t}\right]=I_{t+k-1}$ for $2 \leq k, t \leq n-1$.

(iii) $\left[\sigma_{s_{1}^{m}}, \alpha_{k}\right]=\sigma_{y}$, where $y=s_{k}^{m} x$ and $x \in P_{k+1}$ for $k \geq 2$. Furthermore, $\left[\sigma_{s_{i}^{m}}, \alpha_{k}\right]=\left[\sigma_{s_{i}}, \alpha_{k}\right]^{m}$ for $i, k \geq 2$.

(iv) $\left[\sigma_{s_{i}}, \alpha_{j}^{m}\right]=\sigma_{y}$, where $y=s_{i+j-1}^{m} x$ and $x \in P_{i+j}$ for $i \geq 1$ and $j \geq 2$.

(v) If $i, j \geq 2, \quad\left(p, m_{j-1}\right)=1, \quad m_{j-1} \neq 0 \quad$ and $\left[\sigma_{s_{j-1} m_{j-1} m_{j}}, \ldots, \alpha_{i}\right]=1, \quad$ then $j \geq n-(i-1)$.

(vi) If $i, j \geq 2, m_{j} \neq 0,\left(p, m_{j}\right)=1$ and $\left[\alpha_{n-1}^{m_{n-1}} \alpha_{n-2}^{m_{n-2}} \cdots \alpha_{j}^{m_{j}}, \sigma_{s_{i-1}}\right]=1$, then $j \geq n-(i-1)$.

\section{PROOF.}

(i) This is clear by $\sigma_{s_{t}}^{\alpha_{k}}=\sigma_{s_{t} \alpha_{k}}$.

(ii) This is obvious by (i).

(iii) We have $\left[\sigma_{s_{1}^{m}}, \alpha_{k}\right]=\sigma_{s_{1}^{-m}\left(s_{1} s_{k}\right)^{m}}$. The proof is completed by Lemma 2.6(v).

(iv) We use induction on $m$ and (i).

(v) By (iii), (i) and the fact that $G^{\prime}$ is Abelian, $s_{i+j-2}^{m_{j-1}} s_{i+j-1}^{m_{j}} \cdots \in Z(G)$ for $j \geq 3$, so $i+j-2 \geq n-1$. If $j=2$ then $s_{i}^{m_{1}} x s_{i+1}^{m_{2}} s_{i+2}^{m_{3}} \cdots \in Z(G)$, where $x \in P_{i+1}$ therefore $i \geq n-1$, completing the proof.

(vi) We use (iv) and then proceed as in (v).

THEOREM 3.5. With the notation and assumption of Theorem 3.3, the following results hold:

(i) $\gamma_{j}\left(\operatorname{Aut}^{P_{i}}(G)\right)=I_{(i-1) j+1}$ for $i, j \geq 2$;

(ii) $\operatorname{cl}\left(\operatorname{Aut}^{P_{2}}(G)\right)=n-2$; 
(iii) if $i \geq 3$ and $n=(i-1) c+r(0 \leq r \leq i-2)$, then

$$
\operatorname{cl}\left(\operatorname{Aut}^{P_{i}}(G)\right)= \begin{cases}c-1, & 0 \leq r \leq 1 \\ c, & 2 \leq r \leq i-2\end{cases}
$$

(iv) $\operatorname{Aut}^{P_{i}}(G)$ is Abelian if and only if $i \geq((n+1) / 2)$;

(v) $Z\left(\operatorname{Aut}^{P_{i}}(G)\right)=\operatorname{Aut}^{P_{n-(i-1)}}(G)$ for $2 \leq i \leq((n+1) / 2)$;

(vi) $\zeta_{j}\left(\operatorname{Aut}^{P_{i}}(G)\right)=\operatorname{Aut}^{P_{n-(i-1) j}(G)}$ for $\quad 2 \leq i \leq((n+1) / 2) \quad$ and $\quad 1 \leq j$ $\leq((n-i) /(i-1))$.

\section{PROOF.}

(i) We see that $I_{i}=\left\langle\sigma_{s_{i-1}}, \ldots, \sigma_{s_{n-2}}\right\rangle$ and $\left[I_{i}, \sigma_{s_{1}}\right] \leq I_{i+1}$ for $i \geq 2$. Then Lemma 3.4(ii) implies that $\left[A_{i}, I_{i}\right]=I_{2 i-1}$. Also by Theorem 3.3(ii), we deduce that $\gamma_{2}\left(\operatorname{Aut}^{P_{i}}(G)\right)=I_{2(i-1)+1}$. Now by using induction on $j$ and Lemma 3.4(ii) the result is proved.

(ii) We have $\gamma_{j}\left(\operatorname{Aut}^{P_{2}}(G)\right)=I_{j+1}$ by (i). The result is immediate since $I_{n-1} \neq 1$ and $I_{n}=1$.

(iii) This is evident from (i).

(iv) This is easily proved by considering (iii) and (i).

(v) It is obvious that $\operatorname{Aut}^{P_{n-(i-1)}}(G) \leq Z\left(\operatorname{Aut}^{P_{i}}(G)\right)$ by Theorem 3.3(ii) and 3.4(ii). If $\alpha \sigma_{g} \in Z\left(\operatorname{Aut}^{P_{i}}(G)\right)$ for $\alpha \in A_{i}$ and $g \in P_{i-1}$, then we may write $g=s_{j-1}^{m_{j-1}} \cdots s_{n-1}^{m_{n-1}}$, where $j \geq i, m_{j-1} \neq 0$ and $\left(m_{j-1}, p\right)=1$. Also since $\left|A_{r}: A_{r+1}\right|=p$ for $2 \leq r \leq n-2$, we may write $\alpha=\alpha_{r}^{m_{r}} \cdots \alpha_{n-1}^{m_{n-1}}$, where $r \geq i, m_{r} \neq 0$ and $\left(m_{r}, p\right)=1$. Consequently $\left[\alpha \sigma_{g}, \alpha_{i}\right]=1$ so $\left[\alpha_{i}, \sigma_{g}\right]=1$. Hence, $j \geq n-(i-1)$ by Lemma 3.4(v). Therefore, $\sigma_{g} \in Z\left(\operatorname{Aut}^{P_{i}}(G)\right)$, which implies that $\left[\alpha, \sigma_{s_{i-1}}\right]=1$. This shows that $r \geq n-(i-1)$ by Lemma 3.4(vi), completing the proof.

(vi) $\mathrm{By}(\mathrm{v})$ and Theorem 3.3(iii), $\left|Z\left(\operatorname{Aut}^{P_{i}}(G)\right)\right|=p^{2 i-2}$ for any metabelian $p$-group $G$ of maximal class and order $p^{n}$ with $i \leq((n+1) / 2)$. In what follows we prove, by induction on $j$, that $\zeta_{j}\left(\operatorname{Aut}^{P_{i}}(G)\right)=\operatorname{Aut}^{P_{n-(i-1) j}}(G)$. Suppose that the equality holds for all positive integers less than $j$. We set $H=P_{n-(i-1)}(j-1)$ and observe that

$$
\zeta_{j}\left(\operatorname{Aut}^{P_{i}}(G)\right) / \zeta_{j-1}\left(\operatorname{Aut}^{P_{i}}(G)\right)=Z\left(\operatorname{Aut}^{P_{i}}(G) / \operatorname{Aut}^{H}(G)\right) .
$$

Also $\operatorname{Aut}^{P_{i}}(G) / \operatorname{Aut}^{H}(G) \hookrightarrow \operatorname{Aut}^{P_{i} / H}(G / H)$. Since $G / H$ is a metabelian $p$-group of maximal class and $P_{i}(G / H)=P_{i}(G) / H$, we find that $\left|Z\left(\operatorname{Aut}^{P_{i} / H}(G / H)\right)\right|=p^{2 i-2}$. Then $\left|\operatorname{Aut}^{P_{i} / H}(G / H)\right|=\left|\operatorname{Aut}^{P_{i}}(G) / \operatorname{Aut}^{H}(G)\right|$. Hence $\left|\zeta_{j}\left(\operatorname{Aut}^{P_{i}}(G)\right)\right|=p^{2(i-1) j}$ and $\operatorname{Aut}^{P_{n-(i-1) j}}(G) \leq \zeta_{j}\left(\operatorname{Aut}^{P_{i}}(G)\right)$, completing the proof.

In the rest of this section, we find a necessary and sufficient condition on $G$ for $\left|\operatorname{Aut}_{p}(G): \operatorname{Aut}^{\Phi}(G)\right|=p$. We also give a structure theorem for $\operatorname{Aut}_{p}(G)$. 
THEOREM 3.6. Suppose that $G$ is a metabelian p-group of maximal class of order $p^{n}$, where $p \geq 3$ and $n \geq 2 p-3$. Define the map $\gamma$ by $s^{\gamma}=s s_{1}, s_{1}^{\gamma}=s_{1}$ and $s_{i}^{\gamma}=\left[s_{i-1}^{\gamma}, s^{\gamma}\right]$. Then $\gamma$ extends to an automorphism of $G$ if and only if $s^{p}=\left(s s_{1}\right)^{p}$ and $\left[P_{1}, P_{p-1}\right]=1$.

PROOF. This is obvious when $(p, n)=(3,4)$ so for the rest of the proof suppose that $(p, n) \neq(3,4)$. We first note that if $n \geq 2 p-2$, then $s_{i}^{\gamma}=s_{i}\left[s_{i}, s_{1}\right]^{i-1}\left[s_{i-1}, s_{1}\right]^{i-2}$ $(2 \leq i \leq p-1), s_{p}^{\gamma}=s_{p}\left[s_{p-1}, s_{1}\right]^{p-2}$ and $s_{j}^{\gamma}=s_{j}$ for $j \geq p+1$, by Lemmas 2.12 and 2.4. Now suppose that $\gamma$ is an automorphism. According to Lemma 2.3(ii), we have $s_{1}^{p} s_{p} \in P_{p+1}$ so that $\left[s_{p-1}, s_{1}\right]=1$ since $\left(s_{1}^{p} s_{p}\right)^{\gamma}=s_{1}^{p} s_{p}$. Therefore, $\left[P_{1}, P_{p-1}\right]=1$. On the other hand, $s^{p}=s_{n-1}^{w}$ by Theorem 2.15 and hence $\left(s^{p}\right)^{\gamma}=\left(s_{n-1}^{w}\right)^{\gamma}$, which implies that $s^{p}=\left(s s_{1}\right)^{p}$. Now suppose that $s^{p}=\left(s s_{1}\right)^{p}$ and $\left[P_{1}, P_{p-1}\right]=1$. By using induction on $i$ we may see that $\left[P_{1}, P_{p-i}\right] \leq P_{n-(i-1)}$ $(1 \leq i \leq p-2)$. So by considering the presentation of $G$ given in Theorem 2.15, $k \geq n-p+3$ or equivalently $k \geq p+1$. Finally, we see that $\gamma$ is an automorphism of $G$ by Lemma 2.12(i), (v). Now if $n=2 p-3$, then clearly $p \geq 5$ and so $s_{i}^{\gamma}=s_{i}\left[s_{i}, s_{1}\right]^{i-1}\left[s_{i-1}, s_{1}\right]^{i-2} z$, where $z=\left[\left[s_{2}, s_{1}\right], s_{1}\right]$ and $i \in\{3,4\}$. The value of $\gamma$ on $s_{i}$ for $i \neq 3,4$ is the same as above. Hence, by the same argument we may conclude the result.

THEOREM 3.7. Let $G$ be a metabelian p-group of maximal class of order $p^{n}$. Then $\left|\operatorname{Aut}_{p}(G): \operatorname{Aut}^{\Phi}(G)\right|=p$ if and only if there exists an automorphism of $G$ that maps $s$ to $s s_{1}$ and $s_{1}$ to $s_{1}$.

PROOF. If there exists an automorphism of $G$ that maps $s$ to $s s_{1}$ and $s_{1}$ to $s_{1}$, then $\left|\operatorname{Aut}_{p}(G): \operatorname{Aut}^{\Phi}(G)\right|=p$. Assume that $\left|\operatorname{Aut}_{p}(G): \operatorname{Aut}^{\Phi}(G)\right|=p$, so there exists an automorphism $\alpha$ such that $\alpha \notin \operatorname{Aut}^{\Phi}(G)$. We have $\alpha \in \operatorname{Aut}_{p}^{P_{1}}(G)$ since $\operatorname{Aut}(G) / \operatorname{Aut}^{P_{1}}(G) \hookrightarrow \operatorname{Aut}\left(G / P_{1}\right)$. Hence we may write $s^{\alpha}=s s_{1}^{i} x$ and $s_{1}^{\alpha}=s_{1}^{j+1} y$, where $0 \leq i, j<p$ and $x, y \in \Phi(G)$. We choose $u$ and $w$ in $\Phi(G)$ such that $u^{\alpha}=x$ and $w^{\alpha}=y$. Then by Theorem 3.1 the map $\beta$ defined by $s^{\beta}=s u^{-1}, s_{1}^{\beta}$ $=s_{1} w^{-1}$ is an automorphism of $G$ lying in $\operatorname{Aut}^{\Phi}(G)$. On setting $\delta:=\beta \alpha$, we have $\delta \in \operatorname{Aut}_{p}(G)-\operatorname{Aut}^{\Phi}(G)$ and $s^{\delta}=s s_{1}^{i}, s_{1}^{\delta}=s_{1}^{j+1}$. Now by considering the order of $s_{1}$ and $s_{1}^{\delta}$, we see that $1 \leq j+1<p$ which implies that $(s)^{\delta^{p-1}}=s s_{1}^{t},\left(s_{1}\right)^{\delta^{p-1}}=s_{1} x_{1}$, where $t \neq 0$ and $(t, p)=1$, since $\delta^{p-1} \notin \operatorname{Aut}^{\Phi}(G)$ and $x_{1} \in \Phi(G)$. Now by the same argument as above we obtain an automorphism $\tau$ such that $s^{\tau}=s s_{1}^{t}, s_{1}^{\tau}=s_{1}$ and $\tau \in \operatorname{Aut}_{p}(G)-\operatorname{Aut}^{\Phi}(G)$. So $(s)^{\tau^{m}}=s s_{1}$ and $\left(s_{1}\right)^{\tau^{m}}=s_{1}$, where $m$ is a positive integer satisfying $t m \equiv 1\left(\bmod \left|s_{1}\right|\right)$.

COROLlary 3.8. Let $G$ be a metabelian p-group of maximal class of order $p^{n}$, where $p \geq 3$ and $n \geq 2 p-3$. Then $\left|\operatorname{Aut}_{p}(G): \operatorname{Aut}^{\Phi}(G)\right|=p$ if and only if $\left[P_{1}, P_{p-1}\right]=1$ and $s^{p}=\left(s s_{1}\right)^{p}$. 
LEMMA 3.9. Let $G$ be a metabelian p-group of maximal class of order $p^{n}$, where $p \geq 3$ and $n \geq 2 p-3$. If there exists an automorphism $\gamma$ that maps $s$ to $s s_{1}$ and $s_{1}$ to $s_{1}$, then $\gamma^{p} \in \operatorname{Inn}(G)$ and $\gamma \notin \operatorname{Inn}(G)$.

Proof. We have $\left[P_{1}, P_{p-1}\right]=1$ by Theorem 3.6 so $z=\left[\left[s_{2}, s_{1}\right], s_{1}\right]$ $=1$, by Lemma 2.12(i). On setting $g=s_{1}^{\left(\begin{array}{c}p \\ 2\end{array}\right)} s_{2}^{\left(\begin{array}{c}p \\ 3\end{array}\right)} \cdots s_{p-1}^{\left(\begin{array}{c}p \\ p\end{array}\right)}$, we have $[g, s]$ $=s_{2}^{\left(\begin{array}{c}p \\ 2\end{array}\right)}\left[s_{2}, s_{1}\right]{ }^{\left(\begin{array}{c}m \\ 2\end{array}\right)} s_{3}^{\left(\begin{array}{c}p \\ 3\end{array}\right)} \cdots s_{p}^{\left(\begin{array}{c}p \\ p\end{array}\right)}$, where $m=\left(\begin{array}{c}p \\ 2\end{array}\right)$ since $G^{\prime}$ is Abelian, and by

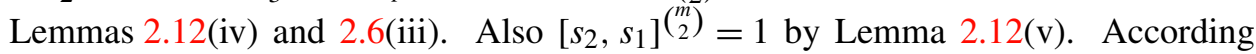
to Lemma 2.14 and Theorem 3.6, $s_{1}^{\left(\begin{array}{c}p \\ 1\end{array}\right)} \cdots s_{p}^{\left(\begin{array}{c}p \\ p\end{array}\right)}=1$ and hence $[s, g]=s_{1}^{p}$. Moreover, $\left[g, s_{1}\right]=1$ by Lemmas 2.6(ii), 2.12(v) and Theorem 3.6. Therefore, $\gamma^{p}=\sigma_{g}$ and obviously $\gamma$ is not an inner automorphism.

THEOREM 3.10. Let $G$ be a metabelian p-group of maximal class of order $p^{n}$, where $p \geq 3$ and $n \geq 2 p-3$. If $\left|\operatorname{Aut}_{p}(G): \operatorname{Aut}^{\Phi}(G)\right|=p$ then $\operatorname{Aut}_{p}(G)=\operatorname{Aut}^{\Phi}(G) \rtimes C_{p}$.

ProOF. By Theorem 3.7, $\gamma$ is an automorphism; and by Lemma 3.9, $\gamma^{p} \in \operatorname{Inn}(G)$. Now following the proof of [8, Proposition 4.1], $G$ can be embedded in a $p$-group $H$ of maximal class of order $p^{n+1}$ and $P_{i}(H)=P_{i-1}(G)$ for $3 \leq i \leq n$. We now choose $t \in H$ such that $t \notin P_{1}(H) \cup G$. But $H$ has positive degree of commutativity by [9, Theorem 3.3.5] and so we deduce that $t^{p} \in P_{n}(H)$, by Lemmas 2.1 and 2.2. Let $\alpha$ be the restriction of $\sigma_{t}$ to $G$; then $\alpha$ has order $p$ since $Z(G)=P_{n}(H)$. We have $\alpha \notin \operatorname{Aut}^{\Phi}(G)$, for otherwise $[G, t] \leq \Phi(G)=P_{2}(G)=P_{3}(H)$. However, $H=\langle G, t\rangle$ would imply that $[H, t] \leq P_{3}(H)$ or equivalently $t \in P_{2}(H)$, which is impossible. Therefore $\operatorname{Aut}_{p}(G)=\operatorname{Aut}^{\Phi}(G) \rtimes\langle\alpha\rangle$, which yields the proof.

REMARK. Let $G$ be a $p$-group of maximal class having order $p^{n}$. In [8, Theorem 4.2] Juhász proves that if $G$ can be embedded in a $p$-group of maximal class, then $\mid$ Aut $_{p}(G):$ Aut $^{\Phi}(G) \mid=p$. We note that the converse of this result is also true when $G$ is metabelian with $n \geq 2 p-3$. Consider the map $\gamma$ defined by $s^{\gamma}=s s_{1}$ and $s_{1}^{\gamma}=s_{1}$. Then $\gamma$ is an automorphism with $\gamma^{p} \in \operatorname{Inn}(G)$ by Theorem 3.7 and Lemma 3.9. Now since $\gamma$ satisfies the conditions of [8, Proposition 4.1], $G$ can be embedded in a $p$ group of maximal class. Notice that our Corollary 3.8 gives another necessary and sufficient condition on $G$ for $\left|\operatorname{Aut}_{p}(G): \operatorname{Aut}^{\Phi}(G)\right|=p$. Juhász also proves that if $G / P_{p+1}(G)$ cannot be embedded in a $p$-group of maximal class and $G$ has positive degree of commutativity, then $\operatorname{Aut}_{p}(G)=\operatorname{Aut}^{\Phi}(G)$. In particular, if $G$ is metabelian, then $\left|\operatorname{Aut}_{p}(G)\right|=p^{2 n-4}$. Finally, we note that the above embedding conditions given by Juhász do not cover the whole class of $p$-groups of maximal class. The following example provides an infinite class of metabelian $p$-groups of maximal class which do not satisfy these conditions. 
EXAMPLE. Suppose that

$$
\begin{gathered}
G \cong\left\langle s, s_{1}, \ldots, s_{n-1}\right| s_{i}=\left[s_{i-1}, s\right],\left[s_{n-1}, s\right]=1,\left[s_{1}, s_{2}\right]=1,\left[s_{i}, s_{j}\right]=1 \\
\left.s^{p}=1, s_{1}^{\left(\begin{array}{c}
p \\
1
\end{array}\right)} \cdots s_{p}^{\left(\begin{array}{c}
p \\
p
\end{array}\right)}=s_{n-1}, s_{i}^{p} s_{i+1}^{\left(\begin{array}{c}
p \\
2
\end{array}\right)} \cdots s_{i+p-1}=1\right\rangle,
\end{gathered}
$$

where $2 \leq i \leq j \leq n-1, n \geq 2 p-3$ and $p \geq 3$. Then $G$ is a $p$-group of maximal class and order $p^{n}$, which cannot be embedded in a $p$-group of maximal class. However, $G / P_{p+1}(G)$ can be embedded in a $p$-group of maximal class.

To prove this, we see that any element $g$ of $G$ may be written as $g=s^{r} s_{1}^{r_{1}} \cdots s_{n-1}^{r_{n-1}}$, where $0 \leq r, r_{1}, \ldots, r_{n-1}<p$. Also $\gamma_{i}(G)=\left\langle s_{i}, \ldots, s_{n-1}\right\rangle, 2 \leq i \leq n-1$, implies that $\operatorname{cl}(G)=n-1$ and $|G|=p^{n}$. By Corollary 3.8, $\left|\operatorname{Aut}_{p}(G): \overline{A u t}^{\bar{\Phi}}(G)\right| \neq p$ since $s^{p} \neq\left(s s_{1}\right)^{p}$. Therefore, by [8, Theorem 4.2], $G$ cannot be embedded in a $p$-group of maximal class. Now by adding the relations $s_{p+1}=\cdots=s_{n-1}=1$ to those of $G$, we find a presentation for $G / P_{p+1}(G)$ in which the relation $s_{1}^{\left(\begin{array}{c}p \\ 1\end{array}\right)} \cdots s_{p}^{\left(\begin{array}{c}p \\ p\end{array}\right)}=1$ holds. On setting $H=G / P_{p+1}(G)$, we see that the map $\gamma$ defined by $s^{\gamma}=s s_{1}$ and $s_{1}^{\gamma}=s_{1}$ is an automorphism of $H$ fixing $s_{i}, 2 \leq i \leq p$. By considering the presentation of $H$, it is easily seen that $\gamma^{p}=\sigma_{g}$, where $g=s_{1}^{\left(\begin{array}{c}p \\ 2\end{array}\right)} \cdots s_{p-1}^{\left(\begin{array}{c}p \\ p\end{array}\right)}$. Hence, by [8, Proposition 4.1], $H$ can be embedded in a $p$-group of maximal class.

\section{The order of $\operatorname{Aut}^{\Phi}(G)$}

In this section we prove that for a noncyclic $p$-group $G$ of maximal class of order $p^{n},\left|\operatorname{Aut}^{\Phi}(G)\right|=p^{2 n-4}$ if and only if $G$ is metabelian. This is evident for the case where $p=2$. Therefore, for the rest of the section we assume that $p$ is an odd prime and $n \geq 4$.

We first give some elementary lemmas.

LEMMA 4.1. Let $G$ be a p-group of maximal class and order $p^{n}$. If $\left[P_{2}(G), P_{2}(G)\right] \leq Z(G)$, then $\left[s_{i}^{-1}, s\right]=s_{i+1}^{-1}\left[s_{i+1}, s_{i}\right](i \geq 2)$.

PROOF. This is immediate by $\left[s_{i} s_{i}^{-1}, s\right]=1$.

LEMMA 4.2. Let $G$ be a p-group of maximal class and order $p^{n},\left[P_{r+1}, P_{r+1}\right]=1$ and $\left[s_{r}, s_{r+1}\right]=z \in Z(G)$ for some $r \geq 2$. Then:

(i) $\left[s_{r}, s_{i}\right]=1$ for $i \geq r+2$;

(ii) $\left[s_{r+1}, s_{r-1}\right]=\left[s_{r-1}, s_{r+1}^{-1}\right]$;

(iii) $\left[\left[s_{r-1}, s_{r}\right], s\right]=\left[s,\left[s_{r}, s_{r-1}\right]\right]$;

(iv) if $\left[s_{r-1}, s_{r}\right]=s_{k}^{a_{k}} s_{k+1}^{a_{k+1}} \cdots s_{n-1}^{a_{n-1}} \quad$ for $\quad k \geq r+2 \quad$ then $\quad\left[s_{r-1}, s_{r+1}\right]$ $=s_{k+1}^{a_{k}} \cdots s_{n-1}^{a_{n-2}} z^{-1}$.

\section{PROOF.}

(i) We use induction on $i(i \geq r+2)$ and the Witt identity.

(ii) This follows from Lemma 2.11 . 
(iii) This is easily seen from Lemma 2.11 and the fact that $\left[s_{r}, s_{r-1}\right] \in P_{r+1}$.

(iv) We have $\left[s_{r-1}, s_{r+1}\right] s_{r+1}^{-1}=s_{r+1}^{-s_{r-1}}=\left[s, s_{r}\right]^{s_{r-1}}=\left[s^{s_{r-1}}, s_{r}^{s_{r-1}}\right]=\left[s\left[s, s_{r-1}\right]\right.$, $\left.s_{r}\left[s_{r}, s_{r-1}\right]\right]=\left[s s_{r}^{-1}, s_{r}\left[s_{r}, s_{r-1}\right]\right]=\left[s, s_{r}\left[s_{r}, s_{r-1}\right]\right]^{s_{r}^{-1}}$ by (i) and the fact that $\left[s_{r}, s_{r-1}\right] \in P_{r+2}$. So $\left[s_{r-1}, s_{r+1}\right] s_{r+1}^{-1}=\left[s,\left[s_{r}, s_{r-1}\right]\right]\left[s, s_{r}\right]^{s_{r}^{-1}}$. Now by (iii), $\left[s_{r-1}, s_{r+1}\right]=\left[\left[s_{r-1}, s_{r}\right], s\right] z^{-1}$ since $\left[s_{r}, s_{r+1}\right]=z$, which completes the proof.

THEOREM 4.3. Let $G$ be a p-group of maximal class of order $p^{n}$, where $n$ is a positive integer. Then $\left|\operatorname{Aut}^{\Phi}(G)\right|=p^{2 n-4}$ if and only if $G$ is metabelian.

Proof. If $G$ is metabelian, then by Theorem 3.1, $\mid$ Aut $^{\Phi}(G) \mid=p^{2 n-4}$. Now suppose that $\mid$ Aut $^{\Phi}(G) \mid=p^{2 n-4}$. By induction on $|G|$ we prove that $G$ is metabelian. If $|G|=p^{4}$ then $\left[P_{2}(G), P_{2}(G)\right] \leq P_{4}(G)=1$ and obviously $G$ is metabelian. Suppose that $|G| \geq p^{5}$ and the theorem is true for each $p$-group of order less than $|G|$. We have $\left|\operatorname{Aut}^{Z}(G)\right|=p^{2}$ by [1, Theorem 1] and the fact that $G$ has no nontrivial Abelian direct factor. Also we have

$$
\operatorname{Aut}^{\Phi}(G) / \operatorname{Aut}^{Z}(G) \hookrightarrow \operatorname{Aut}^{\Phi(G / Z)}(G / Z) .
$$

It follows that $\left|\operatorname{Aut}^{\Phi(G / Z)}(G / Z)\right|=p^{2(n-1)-4}$. Now the group $G / Z$ is metabelian by the induction hypothesis, so $\left[P_{2}(G), P_{2}(G)\right] \leq Z(G)$. By the way of contradiction suppose that $P_{2}(G)$ is not Abelian. Let $r$ be the largest positive integer such that $\left[P_{r}(G), P_{r}(G)\right] \neq 1$, so $\left[P_{r+1}(G), P_{r+1}(G)\right]=1$. We may write $\left[s_{r}, s_{r+1}\right]=z$ $\in Z(G)$ and so, by Lemma 4.2(i), $z \neq 1$. Now the map $\alpha$ defined by $s^{\alpha}=s$ and $s_{1}^{\alpha}=s_{1} s_{2}^{-1}$ is an automorphism of $G$ since $\left|\mathrm{Aut}^{\Phi}(G)\right|=p^{2 n-4}$. By Lemma 4.1 and using induction on $i$, we deduce that $s_{i}^{\alpha}=s_{i} s_{i+1}^{-1}\left[s_{i+1}, s_{i}\right](i \geq 2)$. Therefore, $s_{r}^{\alpha}=s_{r} s_{r+1}^{-1} z^{-1}$ and $s_{k}^{\alpha}=s_{k} s_{k+1}^{-1}(k \geq r+1)$. Also $\left[P_{r}(G), P_{r-1}(G)\right] \leq P_{2 r}(G)$ when $2 r \leq n-1$, since $G / Z$ has positive degree of commutativity. However, $2 r \leq n-1$ always holds, for otherwise $\left[P_{r}(G), P_{r+1}(G)\right] \leq P_{2 r+1}(G)=1$ would imply that $\left[s_{r}, s_{r+1}\right]=1$, a contradiction. Hence, $\left[P_{r}(G), P_{r-1}(G)\right] \leq P_{r+2}(G)$. Therefore, we may write $\left[s_{r-1}, s_{r}\right]=s_{k}^{a_{k}} s_{k+1}^{a_{k+1}} \cdots s_{n-1}^{a_{n-1}}$ when $(k \geq r+2)$. Now since $\alpha$ is an automorphism of $G$, we have $\left[s_{r-1}^{\alpha}, s_{r}^{\alpha}\right]=\left(s_{k}^{\alpha}\right)^{a_{k}} \cdots\left(s_{n-1}^{\alpha}\right)^{a_{n-1}}$. Moreover, $\left[s_{r-1}^{\alpha}, s_{r}^{\alpha}\right]=\left[s_{r+1}, s_{r-1}\right]\left[s_{r-1}, s_{r}\right] z$ by Lemma 4.2(i) and (ii). Also

$$
\left(s_{k}^{\alpha}\right)^{a_{k}} \cdots\left(s_{n-1}^{\alpha}\right)^{a_{n-1}}=\left[s_{r-1}, s_{r}\right]\left(\left[s_{r-1}, s_{r+1}\right] z\right)^{-1}=\left[s_{r-1}, s_{r}\right]\left[s_{r+1}, s_{r-1}\right] z^{-1},
$$

by Lemma 4.2(iv), from which we conclude that $z^{2}=1$, which is impossible.

\section{A structure theorem for $\operatorname{Aut}(G)$ when $p=2,3$}

Let $G$ be a 3-group of maximal class of order $3^{n}$. As in Section 3, it is an easy matter to find $\operatorname{Aut}(G)$ when $n \leq 3$. Therefore, for the rest of this section we assume that $n \geq 4$. When $n=4, G$ is metabelian; and for $n \geq 5, G$ has degree of commutativity $n-4$ by [3, Theorem 3.13] and so is metabelian. 
We deduce the following theorem from Blackburn's observation [3, p. 88] which gives us a presentation for $G$.

THEOREM 5.1. If $G$ is a 3-group of maximal class of order $3^{n}$, then

$$
\begin{gathered}
G \cong\left\langle s, s_{1}, \ldots, s_{n-1}\right| s_{i}=\left[s_{i-1}, s\right],\left[s_{n-1}, s\right]=1,\left[s_{1}, s_{2}\right]=s_{n-1}^{a} \\
\left.s^{3}=s_{n-1}^{b}, s_{1}^{3} s_{2}^{3} s_{3}=s_{n-1}^{c}, s_{i}^{3} s_{i+1}^{3} s_{i+2}=1\right\rangle
\end{gathered}
$$

where $a, b, c \in\{0,1,2\}$ and $2 \leq i \leq n-1$. For $n>4$ there exist three groups which possess no Abelian maximal subgroup, given by $c=0, a=1$ and $b=0,1,2$. If $n$ is even and $n \geq 4$, there exist four groups with an abelian maximal subgroup, given by $a=b=0, c=1,2$ or $a=c=0, b=0,1$. If $n$ is odd and $n>4$ then there exist three groups with an Abelian maximal subgroup, given by $a=b=0, c=1$ or $a=c=0$, $b=0,1$.

By Theorem 3.3 and Corollary 2.10 we obtain the following corollary.

Corollary 5.2. If $G$ is a 3-group of maximal of order $3^{n}$, then $\operatorname{Aut}^{\Phi}(G)$ $=\operatorname{Inn}(G) \rtimes A$, where $A$ is an Abelian subgroup of $\operatorname{Aut}(G)$. Moreover, $A \cong C_{3^{m}}$ $\times C_{3^{m}}$ when $n=2 m+3(m \geq 1)$ and $A \cong C_{3^{m}} \times C_{3^{m+1}}$ when $n=2 m+4(m \geq 0)$.

Corollary 5.3. Let $G$ be a 3-group of maximal class of order $3^{n}$. Then $\mid \operatorname{Aut}_{3}(G)$ : $\operatorname{Aut}^{\Phi}(G) \mid=3$ if and only if $P_{1}$ is Abelian and $\left(s s_{1}\right)^{3}=s^{3}$; in this case $\operatorname{Aut}_{3}(G)$ $\cong \operatorname{Aut}^{\Phi}(G) \rtimes C_{3}$.

PROOF. This follows from Corollary 3.8 and Theorem 3.10.

Now our aim is to find a structure theorem for $\operatorname{Aut}_{2}(G)$, the Sylow 2-subgroup of Aut $(G)$. Since $P_{1}(G)$ and $P_{2}(G)$ are characteristic subgroups of $G, G / P_{2}$ and $P_{1} / P_{2}$ are invariant under $\operatorname{Aut}_{2}(G)$. So by Maschke's theorem there exists $s \in G-P_{1}$ such that $G / P_{2}=P_{1} / P_{2} \oplus\left\langle P_{2}, s\right\rangle / P_{2}$ and $\left\langle P_{2}, s\right\rangle / P_{2}$ is invariant under $\operatorname{Aut}_{2}(G)$. In the rest of this section $s$ will be as above. Therefore, if $\alpha \in \operatorname{Aut}_{2}(G)$ then $s^{\alpha}=s^{i} x$ and $s_{1}^{\alpha}=s_{1}^{j} y$, where $x, y \in P_{2}$ and $i, j \in\{1,-1\}$.

The next lemma follows at once from Theorem 5.1.

LEMMA 5.4. Let $G$ be a 3-group of maximal class of order $3^{n}$. By considering the presentation of $G$ we define the maps $\beta_{j}, j \in\{1,2,3\}$, by $s^{\beta_{1}}=s, s_{1}^{\beta_{1}}=s_{1}^{-1}$, $s^{\beta_{2}}=s^{-1}, s_{1}^{\beta_{2}}=s_{1}, s^{\beta_{3}}=s^{-1}, s_{1}^{\beta_{3}}=s_{1}^{-1}$. Then:

(i) $\quad \beta_{1}$ is an automorphism of $G$ if and only if $P_{1}$ is Abelian and $s^{3}=1$;

(ii) $\beta_{2}$ is an automorphism of $G$ if and only if either $n$ is odd and $s_{1}^{3} s_{2}^{3} s_{3}=1$, or $n$ is even, $P_{1}$ is Abelian and $s^{3}=1$;

(iii) $\beta_{3}$ is an automorphism of $G$ if $n$ is even.

LEMMA 5.5. Let $G$ be a p-group of maximal class of order $p^{n}$ having positive degree of commutativity. If $P_{1}$ is not Abelian then neither is any maximal subgroup of $G$. 
Proof. We have $n>4$ by Theorem 5.1. By way of contradiction, let $M$ be an Abelian maximal subgroup of $G$. Then $M=\langle\Phi(G), y\rangle$, where $y \in G-P_{1}$. So $\mathcal{C}_{G}(y)=\langle y\rangle P_{n-1}$ and $y^{p} \in P_{n-1}$ by Lemmas 2.2 and 2.1. However, $\Phi(G) \leq \mathcal{C}_{G}(y)$ implies that $\left|\mathcal{C}_{G}(y)\right| \geq p^{3}$, which is impossible.

Now by the fact that $\operatorname{Aut}(G) \cong \operatorname{Aut}_{3}(G) \rtimes H$, where $H \leq C_{2} \times C_{2}$, we prove the following theorem.

THEOREM 5.6. Let $G$ be a 3-group of maximal class of order $3^{n}$.

(i) If $P_{1}$ is not Abelian, then $\operatorname{Aut}_{2}(G) \cong C_{2}$.

(ii) If $P_{1}$ is Abelian and $\left(s s_{1}\right)^{3}=s^{3}$, then $\operatorname{Aut}_{2}(G) \cong C_{2} \times C_{2}$ when $|s|=3$ and $\operatorname{Aut}_{2}(G) \cong C_{2}$ when $|s|=9$.

(iii) If $P_{1}$ is Abelian and $\left(s s_{1}\right)^{3} \neq s^{3}$, then $\operatorname{Aut}_{2}(G) \cong C_{2} \times C_{2}$ when $n$ is even and $\operatorname{Aut}_{2}(G) \cong C_{2}$ when $n$ is odd.

Proof. (i) According to Theorem 5.1 and Lemmas 5.4 and 5.5, $A_{4} t_{2}(G)=\left\langle\beta_{2}\right\rangle$ if $n$ is odd and $\operatorname{Aut}_{2}(G)=\left\langle\beta_{3}\right\rangle$ if $n$ is even.

(ii) By Lemma 5.4, we have $\operatorname{Aut}_{2}(G)=\left\langle\beta_{1}\right\rangle \times\left\langle\beta_{2}\right\rangle$ when $|s|=3$. Now if $|s|=9$, by Lemma 5.4, we have $\operatorname{Aut}_{2}(G)=\left\langle\beta_{2}\right\rangle$ or $\operatorname{Aut}_{2}(G)=\left\langle\beta_{3}\right\rangle$ depending on the parity of $n$.

(iii) By considering Theorem 5.1, we see that $b=0$ and so $\operatorname{Aut}_{2}(G)=\left\langle\beta_{1}\right\rangle \times\left\langle\beta_{2}\right\rangle$ by Lemma 5.4, when $n$ is even. Now if $n$ is odd, by Theorem 5.1 and Lemma 5.4, we have $\operatorname{Aut}_{2}(G)=\left\langle\beta_{1}\right\rangle$.

LEMMA 5.7. Let $G$ be a 3-group of maximal class of order $3^{n}$. Then every element out of $P_{1}$ has order 3 or 9 . Furthermore, when $P_{1}$ is Abelian, all elements out of $P_{1}$ have the same order if and only if $\left(s s_{1}\right)^{3}=s^{3}$.

Proof. According to Lemma 2.2, every element out of $P_{1}$ has order 3 or 9 . We have $\left(s s_{1}\right)^{3}=s^{3} s_{1}^{3} s_{2}^{3} s_{3}$ when $P_{1}$ is Abelian. If $\left(s s_{1}\right)^{3}=s^{3}$ then $c=0$ by Theorem 5.1. Also any element out of $P_{1}$ has the form $s^{t} s_{1}^{t_{1}} \cdots s_{n-1}^{t_{n-1}}$, where $0<t<3$ and $0 \leq t_{i}<3$. Therefore by [3, Equation 40] $\left(s^{t} s_{1}^{t_{1}} \cdots s_{n-1}^{t_{n-1}}\right)^{3}=s^{3 t}$, completing the proof. Now suppose that all elements out of $P_{1}$ have the same order. If $c=0$ then $\left(s s_{1}\right)^{3}=s^{3}$, and if $c \neq 0$ then $b=0$ or equivalently $s^{3}=1$ by Theorem 5.1 . Hence $\left(s s_{1}\right)^{3}=1$, as desired.

THEOREM 5.8. Let $G$ be a 3-group of maximal class of order $3^{n}$. If $G$ has no Abelian maximal subgroup, then $\operatorname{Aut}(G) \cong \operatorname{Aut}^{\Phi}(G) \rtimes C_{2}$. If $G$ has an Abelian maximal subgroup, then $P_{1}$ is Abelian and every element out of $P_{1}$ has order 3 or 9.

(i) If all elements out of $P_{1}$ have order 3 then

$$
\operatorname{Aut}(G) \cong\left(\operatorname{Aut}^{\Phi}(G) \rtimes C_{3}\right) \rtimes\left(C_{2} \times C_{2}\right),
$$

and if all elements out of $P_{1}$ have order 9 then

$$
\operatorname{Aut}(G) \cong\left(\operatorname{Aut}^{\Phi}(G) \rtimes C_{3}\right) \rtimes C_{2} .
$$


(ii) Suppose that elements out of $P_{1}$ do not have the same order. If $n$ is even then $\operatorname{Aut}(G) \cong \operatorname{Aut}^{\Phi}(G) \rtimes\left(C_{2} \times C_{2}\right)$, and if $n$ is odd then

$$
\operatorname{Aut}(G) \cong \operatorname{Aut}^{\Phi}(G) \rtimes C_{2} .
$$

PROOF. This is a straightforward consequence of the lemmas above.

Now if $G$ is a 2-group of maximal class, we may deduce some parts of the following theorem from the result of Section 3; however, there is also a simple proof as shown below.

THEOREM 5.9. Let $G$ be a 2-group of maximal class of order $2^{n}(n \geq 3)$. If $G$ is the dihedral group of order $2^{n}$ or the quaternion group of order $2^{n}$, then $\operatorname{Aut}^{\Phi}(G)$ $\cong \operatorname{Inn}(G) \rtimes C_{2^{n-3}}$ and $\operatorname{Aut}(G) \cong \operatorname{Aut}^{\Phi}(G) \rtimes C_{2}$. If $G$ is the semi-dihedral group of order $2^{n}$, then $\operatorname{Aut}(G)=\operatorname{Aut}^{\Phi}(G) \cong \operatorname{Inn}(G) \rtimes C_{2^{n-3}}$.

PROOF. We know that

$$
\begin{aligned}
& D_{2^{n}} \cong\left\langle x, y \mid x^{2^{n-1}}=y^{2}=(x y)^{2}=1\right\rangle, \\
& Q_{2^{n}} \cong\left\langle x, y \mid x^{2^{n-2}}=y^{2}, y^{-1} x y=x^{-1}, x^{2^{n-1}}=1\right\rangle \text { and } \\
& S D_{2^{n}} \cong\left\langle x, y \mid x^{2^{n-1}}=y^{2}=1, y^{-1} x y=x^{-1+2^{n-2}}\right\rangle .
\end{aligned}
$$

If $G$ is $D_{2^{n}}$ or $Q_{2^{n}}$, then there are automorphisms $\alpha, \beta, \gamma$ and $\delta$ defined by $x^{\alpha}=x^{-1}$, $y^{\alpha}=y, x^{\beta}=x, y^{\beta}=x^{2} y, x^{\gamma}=x^{5}, y^{\gamma}=y$ and $x^{\delta}=x^{-1}, y^{\delta}=x^{-1} y$. It is then easy to check that $\operatorname{Inn}(G)=\langle\alpha, \beta\rangle,|\gamma|=2^{n-3}, \delta \notin \operatorname{Aut}^{\Phi}(G)$ and $|\delta|=2$. If $G=S D_{2^{n}}$, there are automorphisms $\alpha, \beta$ and $\gamma$ defined by $x^{\alpha}=x^{-1+2^{n-2}}, y^{\alpha}=y, x^{\beta}=x$, $y^{\beta}=x^{-2+2^{n-2}} y$ and $x^{\gamma}=x^{5}, y^{\gamma}=y$. Hence $\operatorname{Inn}(G)=\langle\alpha, \beta\rangle$ and $|\gamma|=2^{n-3}$ and the rest is clear.

\section{Acknowledgement}

This work was carried out under the supervision of Professor M. F. Newman during our visit to the Australian National University. We are deeply indebted to Professor Newman for setting up the problem, showing us how to find helpful examples, reading and correcting the first version of the manuscript and supervising our work kindly.

\section{References}

[1] J. E. Adney and T. Yen, 'Automorphisms of a p-group', Illinois J. Math. 9 (1965), 137-143.

[2] A. H. Baartmans and J. J. Woeppel, 'The automorphism group of a $p$-group of maximal class with an Abelian maximal subgroup', Fund. Math. 93(1) (1976), 41-46.

[3] N. Blackburn, 'On a special class of p-groups', Acta Math. 100 (1958), 45-92.

[4] A. Caranti and S. Mattarei, 'Automorphisms of p-groups of maximal class', Rend. Sem. Mat. Univ. Padova 115 (2006), 189-198.

[5] A. Caranti and C. M. Scoppola, 'A remark on the orders of $p$-groups that are automorphism groups', Boll. Unione Mat. Ital. A (7) 4(2) (1990), 201-207. 
[6] A. Caranti and C. M. Scoppola, 'Endomorphisms of two-generated metabelian groups that induce the identity modulo the derived subgroup', Arch. Math. 56(3) (1991), 218-227.

[7] B. Huppert, Endliche gruppen, Vol. 1 (Springer, Berlin, 1967).

[8] A. Juhász, 'The group of automorphisms of a class of finite p-groups', Trans. Amer. Math. Soc. 270(2) (1982), 469-481.

[9] C. R. Leedham-Green and S. McKay, The structure of groups of prime power order (Oxford University Press, Oxford, 2002).

[10] I. Malinowska, 'Finite $p$-groups with few $p$-automorphisms', J. Group Theory 4 (2001), 395-400.

\section{S. FOULADI, Faculty of Mathematics, University for Teacher Education,} 599 Taleghani Avenue, Tehran 15618, Iran

e-mail: s_fouladi@tmu.ac.ir

R. ORFI, Faculty of Mathematics, University for Teacher Education, 599 Taleghani Avenue, Tehran 15618, Iran

e-mail: r_orfi@tmu.ac.ir 